\title{
Humidity-Sensitive Properties of Polyelectrolytes Containing Alkoxysilane Crosslinkers
}

\author{
Chil-Won Lee, Hyung-Seok Park, and Myoung-Seon Gong* \\ Department of Chemistry, Dankook University, Cheonan, Chungnam 330-714, Korea
}

Received March 10, 2004; Revised May 6, 2004

\begin{abstract}
We have prepared new polyelectrolytes containing trialkoxysilyl groups by copolymerizing 3-(trimethoxysilyl)propyl methacrylate (TSPM) with either [2-(methacryloyloxy)ethyl]trimethyl ammonium chloride (METAC), [2(methacryloyloxy)ethyl]dimethyl propyl ammonium bromide (MEDPAB), or [2-(acryloyloxy)ethyl]trimethyl ammonium chloride (AETAC). The copolymers TSPM/METAC, TSPM/MEDPAB, and TSPM/METAC having compositions of 15/85, 10/90, and 5/95, respectively, were self-crosslinkable polyelectrolytes that possess humidity-sensitive properties. We measured the impedances of the copolymers at various relative humidities (RHs) and found that the resistance was dependent on the content of METAC, MEDPAB, or AETAC. The impedance changed from $10^{7} \Omega$ at $20 \%$ RH to $10^{3} \Omega$ at $95 \% \mathrm{RH}$, which is quite a suitable range for a humidity sensor that is to be utilized at ambient humidity. We also performed tests of the materials temperature dependence, hysteresis, response time, and water durability.
\end{abstract}

Keywords: humidity sensor, polyelectrolytes, alkoxysilane crosslinking.

\section{Introduction}

Recently, the importance of humidity sensors utilizing polymer film has been growing. ${ }^{1}$ Various types of humiditysensitive monomers in the form of cation or anion salts have been used for humidity sensing materials. ${ }^{2,3}$ The chemical structures of base polymer determines sensor's sensitivity, stability, reliability, and electrical characteristics.

The typical coupling agents, such as 3-(trimethoxysilyl) propyl methacrylate, are difunctional molecule having both the vinyl group which reacts with the resins and the alkoxysilyl groups that interact with silica or alumina. The treatment with these agents could form covalent bonding to improve the adhesion between incompatible inorganic surface and/or organic polymers through intra- or intermolecular reaction. Generally, the water resistant properties tend to improve when the crosslinking of polyelectrolyte is increased. ${ }^{4,5}$

The humidity sensors made of polyelectrolyte shows a poor durability against water or dew, because they are generally soluble in water. Various methods have been attempted to improve the performance of a humidity sensor at high humidity. ${ }^{6-17}$ Cross-linking and copolymerization with hydrophobic monomer have been frequently employed for the preparation of water-resistant polymer membrane..$^{11,18-23}$

*e-mail: msgong@dankook.ac.kr

1598-5032/06/311-05@2004 Polymer Society of Korea
We have been interested in the synthesis of a new family of organic-inorganic chemical hybrid materials in which the vinyl polymer chains are uniformly distributed by covalently binding to the inorganic networks at the molecular level via the sol-gel process. ${ }^{24}$

In this article we present a new class of organic-inorganic hybrid polyelectrolytes for humidity-sensitive materials. The polyelectrolytes were derived from the acid-catalyzed sol-gel reactions of inorganic precursor, with various amounts of polymer precursor (i.e. quaternary ammonium salt-containing reactive trialkoxysilyl functional groups). The Impedance characteristics of the humidity-sensitive films were examined and evaluated as a function of the relative humidity.

\section{Experimental}

Chemicals and Instrument. [2-(Methacryloyloxy)ethyl] trimethyl ammonium chloride (METAC) and [2-(acryloyloxy) ethyl] trimethyl ammonium chloride (AETAC) were purified after evaporation of water. 3-(Trimethoxysilyl)propyl methacrylate (TSPM) was used as received. [2-(Methacryloyloxy) ethyl]dimethyl propyl ammonium bromide (MEDPAB) was prepared by the method previously reported. ${ }^{25}$ 2-Methoxyethanol was purified by conventional purification methods. Dimethylsulfoxide (DMSO) was purified by distillation under reduced pressure after drying by refluxing a mixture of DMSO and benzene using Dean-Stark separator.

The humidity and temperature controller (Jeio Tech Korea, 
Model TM-NFM-L; 20\%95\%RH) was used for the measurement of relative humidity at constant temperature. The impedance of the sensors was measured with a LCR meter

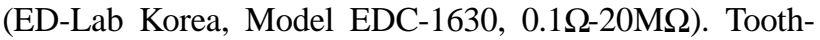
comb gold electrode (width: $0.15 \mathrm{~mm}$; thickness of electrode: $8 \sim 10 \mu \mathrm{m})$ was silkscreen-printed on the alumina substrate $(10 \mathrm{~mm} \times 5.08 \mathrm{~mm} \times 0.635 \mathrm{~mm})$ using a 280 -mesh sieve. A soldering pad and over-coat were formed using silver-palladium alloy and glass paste, respectively. The surface resistance of the gold electrode was found to be less than $0.04 \Omega$ The sensor chips were rinsed in $0.1 \mathrm{~N} \mathrm{HCl}$ for $3 \mathrm{~h}$ and washed with distilled water before use.

Representative Preparation of Self-Crosslinkable Humidity-Sensitive Copolymers. A mixture of the humidity sensitive monomer METAC (18.63 g, $90 \mathrm{mmol}$ ), comonomer TSPM (2.48 g, $10 \mathrm{mmol})$ and AIBN (0.18 g, $0.108 \mathrm{mmol})$ dissolved in anhydrous 2-methoxyethanol $(60 \mathrm{~g})$ were placed in a glass ampoule. The solution was degassed by freeze-thaw method. The sealed glass ampoule was heated at $60^{\circ} \mathrm{C}$ and maintained for $24 \mathrm{~h}$. The polymerized mixture was precipitated into a large amount of anhydrous ethyl ether. The product was purified by dissolution in dry 2-methoxyethanol followed by reprecipitation in $n$-hexane. The copolymers were dried under vacuum at $50^{\circ} \mathrm{C}$ for $12 \mathrm{~h}$. Other copolymers of TSPM with MEDPAB and AETAC were prepared by the similar methods described above.

Fabrication of Humid Membrane. The copolymer $(0.65$ $\mathrm{g}), 0.1 \mathrm{~N} \mathrm{HCl}(0.01 \mathrm{~g})$ and water $(0.5 \mathrm{~g})$ were dissolved in anhydrous DMSO $(5.0 \mathrm{~g})$ at room temperature. The mixture was coated on the alumina electrode by dipping. After the sensor chips were heated to induce the cross-linking reaction at $60^{\circ} \mathrm{C}$ for $2 \mathrm{~h}$ and at $120^{\circ} \mathrm{C}$ for $6 \mathrm{~h}$, They were finally rinsed with ethanol/water $(90 / 10)$ at $50^{\circ} \mathrm{C}$ for $5 \mathrm{~h}$ and dried under vacuum at $50^{\circ} \mathrm{C}$ for $12 \mathrm{~h}$.

Measurements of Impedance Characteristics. Impedance characteristics of the sensor were measured as a function of relative humidity for dessication and humidification processes, relative humidity varied from $20 \% \mathrm{RH}$ to $95 \% \mathrm{RH}$ at $25^{\circ} \mathrm{C}$. The impedance was measured at $1 \mathrm{~V}$ and $1 \mathrm{kHz}$. The impedance was also measured at 15,25 and $35^{\circ} \mathrm{C}$. Frequency dependence was obtained by changing frequencies at $100 \mathrm{~Hz}, 1 \mathrm{kHz}$, and $10 \mathrm{kHz}$. Response time was determined at $33 \% \mathrm{RH}$ and $94 \% \mathrm{RH}$ prepared by saturated salt solution of $\mathrm{KNO}_{3}$ and $\mathrm{MgCl}_{2} \cdot 6 \mathrm{H}_{2} \mathrm{O}$, respectively.

\section{Results and Discussion}

Trimethoxysilyl-functionalized polyelectrolytes precursors were prepared by free radical copolymerization of TSPM with METAC, MEDPAB and AETAC using AIBN as an initiator in 2-methoxyethanol at $60^{\circ} \mathrm{C}$ as shown in Scheme I. The copolymers were hygroscopic and very sensitive to water, which resulted in the crosslinking of copolymers. The copolymers were soluble in common organic solvents such

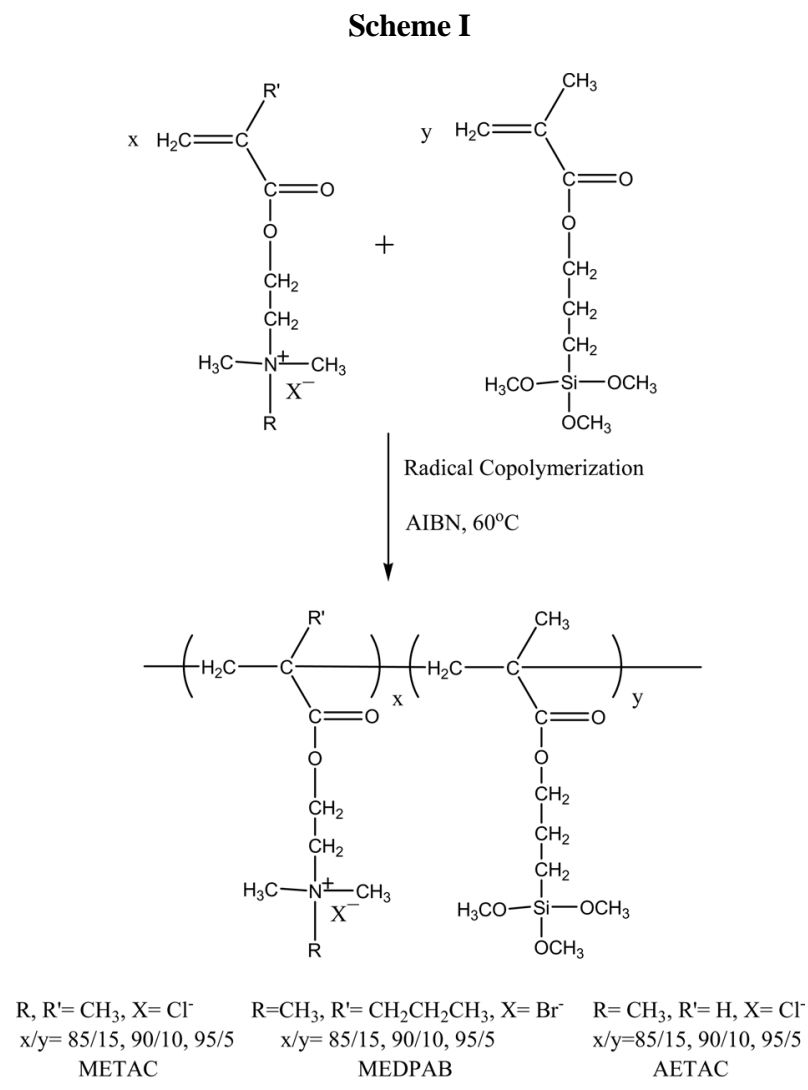

as ethanol, 2-methoxyethanol, DMSO and $\mathrm{N}$-methylpyrrolidinone. The chemical structures and compositions of the polyelectrolyte precursors were characterized by ${ }^{1} \mathrm{H}$ NMR and FT IR spectroscopy.

The comonomer, TSPM was adopted for both crosslinking and anchoring of polyelectrolytes to the alumina substrate as shown in Scheme II. The copolymer precursor could be hydrolyzed in the presence of $\mathrm{HCl}$ as catalyst to yield hybrid materials. When $15 \mathrm{~mol} \%$ of TSPM was used, the resulting polyelectrolyte was stable enough to endure high humidity or a dew point. Higher TSPM content in the precursor should lead to greater extent of crosslinking of the polyelectrolyte chain. When the solution of copolymer was coated on the electrode, the adhesion property to alumina electrode was very excellent.

Impedance versus relative humidity characteristics of the sensors were measured as a function of relative humidity in the range of 20 to $95 \% \mathrm{RH}$ at $1 \mathrm{~V}$ and $1 \mathrm{kHz}$. The typical impedance characteristic curves of the crosslinked copolymers at $25^{\circ} \mathrm{C}$ are shown in Figure 1. In the case of TSPM/METAC and TSPM/MEDPAB $=10 / 90$, the average impedance at $30 \sim 90 \%$ RH varied from $645 \mathrm{k} \Omega$ to $880 \Omega$ and from $5.45 \mathrm{M} \Omega$ to $1270 \Omega$ respectively. For the device fabricated with TSPM/MEDPAB $=10 / 90$ cross-linked by acid catalyst, it decreased by 4 orders of magnitude from $10^{7}$ to $10^{3}$ from 20 to $95 \% \mathrm{RH}$. The semi-logarithmic response curve 


\section{Scheme II}

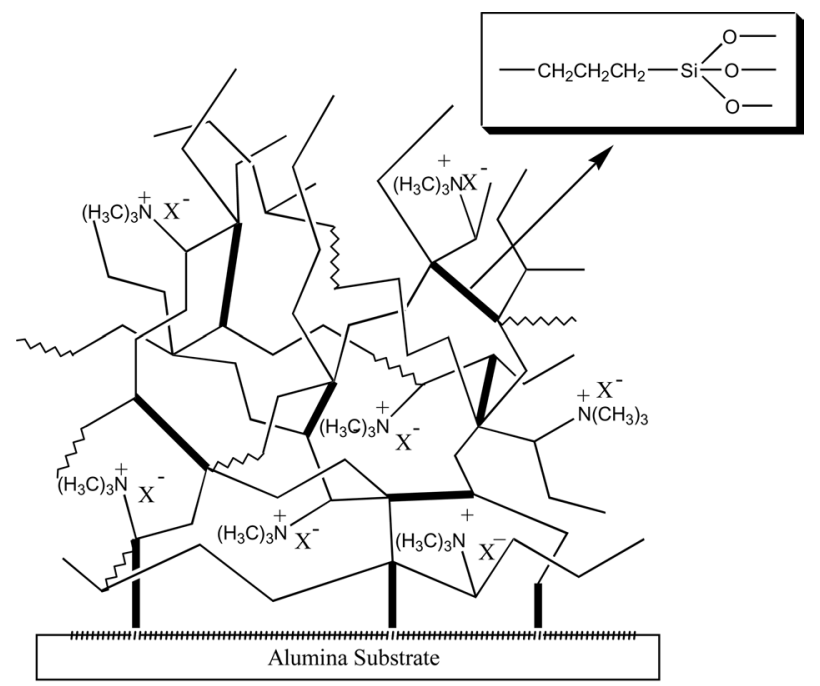

has a moderate linearity over all humidity regions.

The copolymer derived from TSPM/MEDPAB $=10 / 90$ showed a good adhesion to alumina substrate. Impedance of the copolymers increased gradually with an increase in the content of comonomer TSPM.

The hysteresis of humidity sensors with TSPM/MEDPAB $=10 / 90$ between humidification process and desiccation process was also measured between $20 \% \mathrm{RH}$ and $95 \% \mathrm{RH}$ as shown in Figure 1. The impedance on desorption was slightly lower than that on absorption and the corresponding hysteresis fell within $1.5 \% \mathrm{RH}$. This fact implies that the rate of absorption and desorption during the desiccation process of the absorbed water is slower than that during the humidification process.

The polymers were cured in the presence of acid catalyst using sol-gel process. When we used different amounts of acid catalyst, the change of impedances was negligible as shown in Figure 2. The volume of acid catalyst was set at $2.0 \mathrm{~mL}$. The alkoxysilane-crosslinking reactions proceeded completely with $0.1 \mathrm{~N} \mathrm{HCl}$.

To determine the temperature effects, the humidity sensor element was measured at three different temperatures of 15 , 25 or $35^{\circ} \mathrm{C}$. Figure 3 shows the corresponding humidityimpedance characteristics of the sensor element. The impedances of sensor across the full humidity range generally decreased with an increase of the temperature because the mobility of carrier ion might be improved. The temperature dependence was observed for the humidity range studied. The temperature dependence coefficients between $15 \sim 25^{\circ} \mathrm{C}$ (Figure 3; a, c, e etc.) and between $25 \sim 35^{\circ} \mathrm{C}$ (Figure 3; b, d, $f$ etc.) were $-0.50 \sim-0.67 \% \mathrm{RH} /$ and $-0.45 \sim-0.62 \% \mathrm{RH} /$, respectively. At a high humidity and at low temperature, the temperature coefficient became smaller. The influence of the temperature on the sensor characteristics could be com-

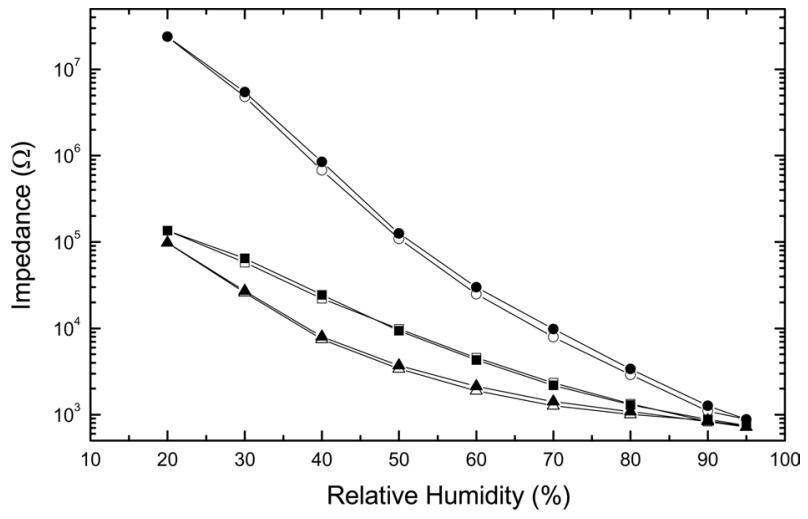

Figure 1. Dependence of impedance on the relative humidity and hysteresis for the humidity sensor using $(0)$ TSPM/MEDPAB $=$ 10/90 (ם) TSPM/METAC $=10 / 90$, and $(\boldsymbol{\Delta})$ TSPM/AETAC $=$ $10 / 90$ at $1 \mathrm{kHz}$ at $25^{\circ} \mathrm{C}$ and $1 \mathrm{~V}$; (solid symbol) absorption and (open symbol) desorption.

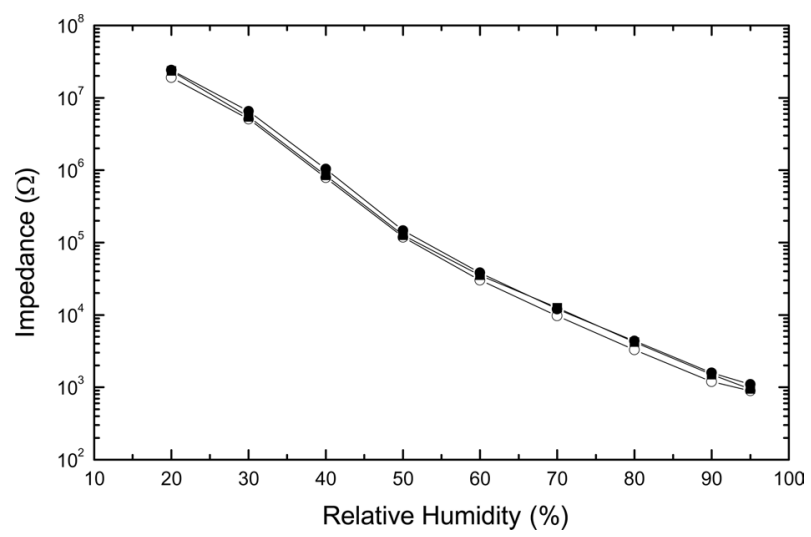

Figure 2. The effect of acid catalyst on the impedance of the polyelectrolyte of TSPM/MEDPAB = 10/90; (O) $0.1 \mathrm{~N} \mathrm{HCl}$, (ם) $0.5 \mathrm{~N} \mathrm{HCl}$ and $(\boldsymbol{O}) 1 \mathrm{~N} \mathrm{HCl}$.

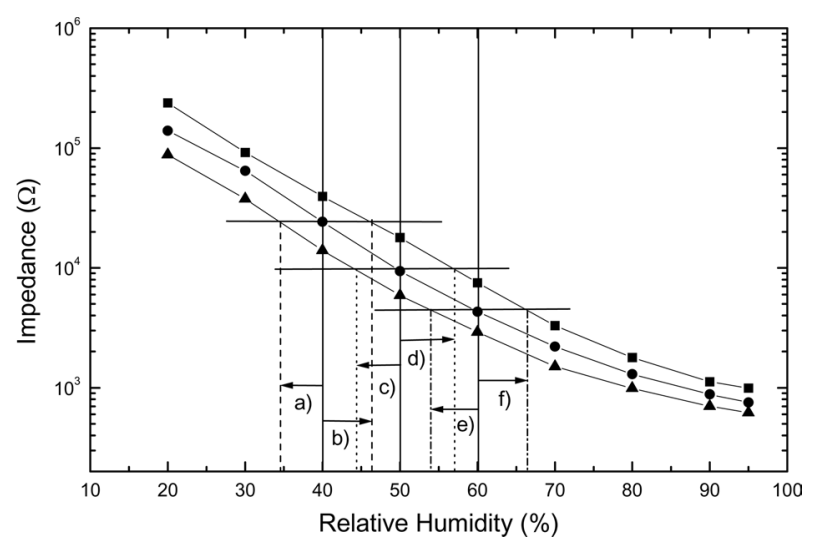

Figure 3. The impedance dependence on relative humidity of humidity sensor obtained from TSPM/METAC $=10 / 90$ at $15^{\circ} \mathrm{C},(\bigcirc) 25^{\circ} \mathrm{C}$ and $(\Delta) 35^{\circ} \mathrm{C}$ at $1 \mathrm{kHz}$ and $1 \mathrm{~V}$. 


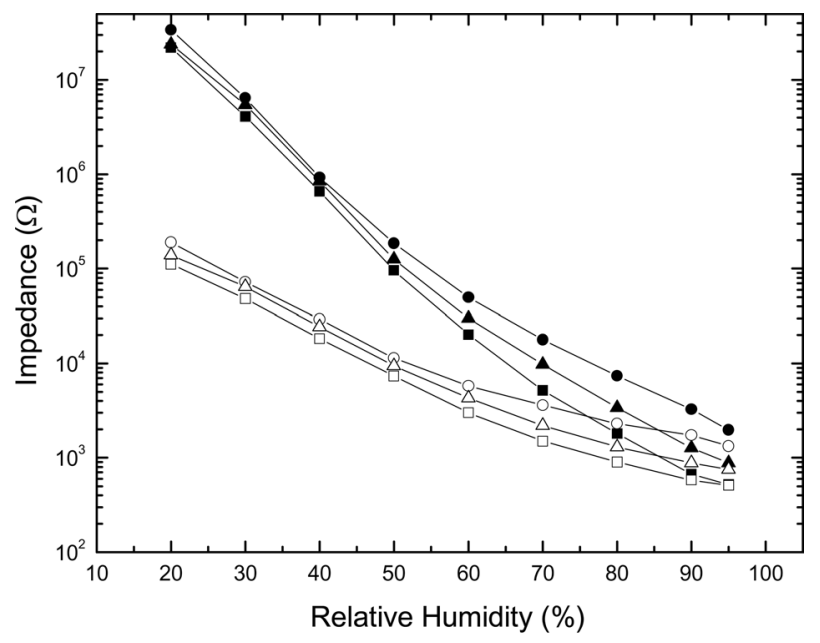

Figure 4. The impedance dependence on the applied frequency of (○) $1 \mathrm{kHz},(\boldsymbol{\Delta}) 5 \mathrm{kHz}$ and $(\boldsymbol{\square}) 10 \mathrm{kHz}$ (TSPM/MEDPAB= $10 / 90)$, and $(\bigcirc) 1 \mathrm{kHz},(\triangle) 5 \mathrm{kHz}$ and $(\square) 10 \mathrm{kHz}$ (TSPM/ $\operatorname{METAC}=10 / 90)$ at $25^{\circ} \mathrm{C}$ and $1 \mathrm{~V}$.

pensated by integrating a NTC resister for the application of a humidity sensor.

The electrical characteristics of the polymeric film humidity sensor were measured in a.c. fields, because the sensor became unstable under d.c. fields due to the electrolysis of the polyelectrolyte. The conducting mechanism of this sensor was ionic with protons as main charge carriers. The impedance of the sensor was affected by the frequency of the applied a.c. voltage. Figure 4 shows the frequency dependence of the resistance of sensor at $100 \mathrm{~Hz}, 1 \mathrm{kHz}$ and $10 \mathrm{kHz}$. The frequency characteristics of the sensor were also dependent on the relative humidity; at low humidity the sensor resistance was more affected by the frequency.

The response times of the sensor were measured when the sensor was applied by 1 voltage at $1 \mathrm{kHz}$. This sensor was moved very quickly from a humidity level of $94 \% \mathrm{RH}$ (sensor being kept in a closed bottle saturated with water vapor) to another bottle adjusted to a humidity level of $33 \% \mathrm{RH}$, and vice versa. Figure 5 shows the humidity response curve corresponding to water adsorption and desorption. A relatively long time seems to be required to desorb the water vapor. The typical response times were measured to be approximately $105 \mathrm{~s}$ for adsorption process and $115 \mathrm{~s}$ for desorption processes. A substantial rise in the first $50 \mathrm{~s}$ period, followed by a more gradual rise until the sensor equilibrated at $94 \% \mathrm{RH}$. Returning the sensor to $33 \% \mathrm{RH}$, the impedance returned to the original $33 \% \mathrm{RH}$ base film value. Repeated cycling of the sensor between those two humidity values gave almost superimposable response curve.

The long-term stability of the humidity sensor in water was evaluated. Each sensor was immersed in water for certain period and then dried in air. These procedures were repeated several times.

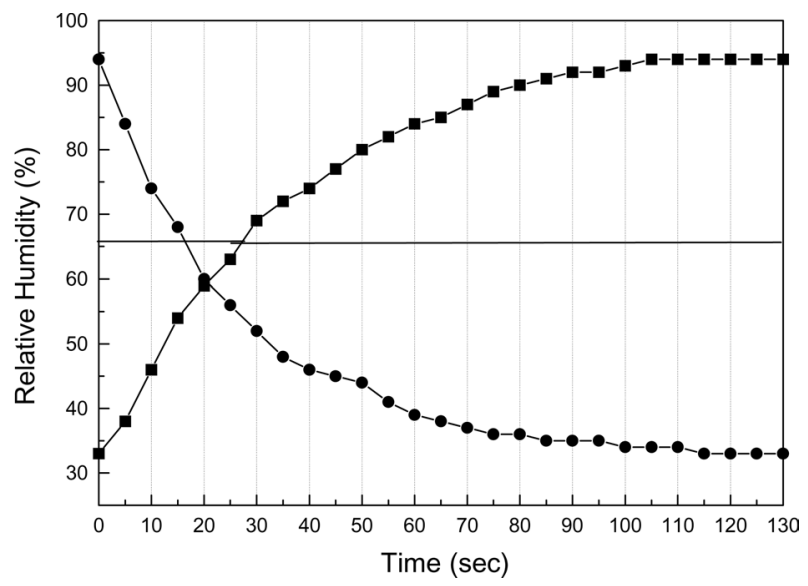

Figure 5. Response time of the humidity sensor obtained from MEDPAB/TSPM = 90/10; ( $\mathbf{\square})$ absorption and ( $)$ desorption process at $25^{\circ} \mathrm{C}$.

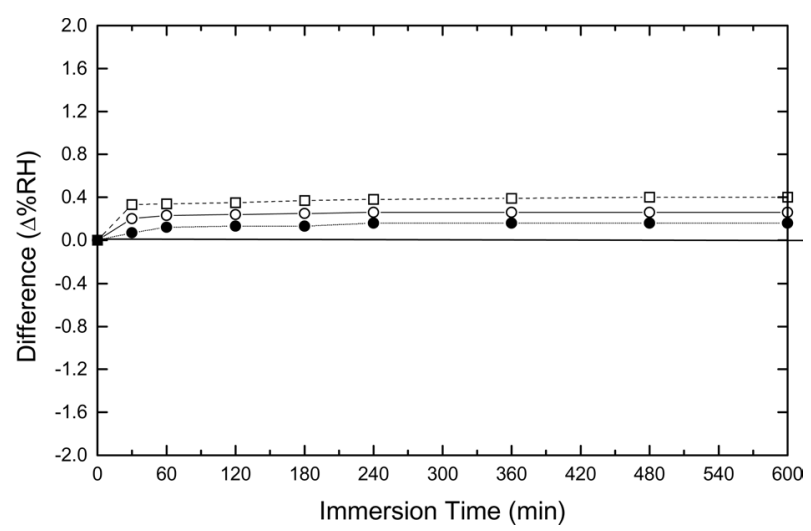

Figure 6. Durability test (measured at $70 \% \mathrm{RH}$ ) of humidity sensors of TSPM/MEDPAB =

which were soaked in water.

The alkoxysilane-crosslinked polyelectrolyte film humidity sensors were durable against water because of being insoluble in water. The increments of impedance were as small as $+0.4 \%$ RH after soaking in water as shown in Figure 6. This initial change may be caused by washing-away of the insufficiently crosslinked copolymer in water. Based on the result, it was found that crosslinked polyelectrolytes using crosslinking of reactive alkoxysilane copolymers were very efficient to improve water durability.

\section{Conclusion}

A new family of polyelectrolytes containing silane crosslinking group, potentially useful in humidity sensitive materials, was synthesized through the $\mathrm{HCl}$-catalyzed sol-gel reactions of copolymer precursor containing reactive alkoxysilane unit at various composition. Humidity sensor using 
METAC, MEDPAB and AETAC copolymers showed the impedance varying from $10^{3} \Omega$ to $10^{7} \Omega$ in the humidity range from $20 \% \mathrm{RH}$ to $95 \% \mathrm{RH}$. Temperature coefficient between $15 \sim 35$ is $-0.45 \sim-0.67 \% \mathrm{RH} /$ and the response time was 115 s between $33 \% \mathrm{RH}$ and $94 \% \mathrm{RH}$. The cross-linking was efficient in improving water durability and this type of humidity sensor showed long-term stability at high temperature and high humidity and could be considered applicable as a humidity sensor.

Acknowledgements. This work was supported by grant No. 01-2002-000-00378-0 from the Basic Research Program of Korea Science \& Engineering Foundation.

\section{References}

(1) Handbook of Materials and Moisture, Kyoritsu Publish Co., Japan, 1991, pp. 151-167.

(2) Y. Sakai, Y. Sadaoka, and M. Matsuguchi, Sens. Actuators B, 85, 35 (1996).

(3) M. Hijikigawa, S. Miyosh, T. Sugihara, and A. Jinda, Sens. Actuators, 4, 307 (1983).

(4) Y. Wei, D. Jin, G. Wei, D. Yang, and J. Xu, J. Appl. Polym. Sci. 70, 1689 (1998).

(5) Y. Wei, W. Wang, J. M. Yeh, B. Wang, D. Yang, J. K. Murray, Jr., D. Jin, and G. Wei, Hybrid Organic-Inorganic Composite, J. E. Mark, C. Y. C. Lee and V. A. Bianconi, Eds., ACS. Symp. Ser. 585, American Chemical Society, Washington DC, 1995, p125.

(6) Y. Sakai, Y. Sadaoka and K. Ikeuchi, Sens. Actuators, 9, 125 (1986).

(7) Y. Sakai, Y. Sadaoka, M. Matsuguchi, Y. Kanakura, and M. Tamura, J. Electrochem. Soc., 138, 2474 (1991).
(8) C. W. Lee, H. W. Rhee, and M. S. Gong, Sens. Actuators B, 73, 124 (2001).

(9) Y. Sakai, M. Matsuguchi, Y. Sadaoka, and K. Hirayama, J. Electrochem. Soc., 140, 432 (1993).

(10) Y. Sakai, Y. Sadaoka, and M. Shimada, Sens. Actuators B, 16, 359 (1989).

(11) H. W. Rhee, M. H. Lee, and M. S. Gong, Sens. Actuators B, 73, 185 (2001).

(12) C. W. Lee, H. W. Rhee, and M. S. Gong, Syn. Met., 106, 177 (1999).

(13) J. S. Jo, H. M. Lee, R. Y. Lee, K. H. Kim and M. S. Gong, Polymer (Korea), 16, 266 (1992).

(14) J. S. Paek, R. Y. Lee, J. K. Park, and M. S. Gong, Polymer (Korea), 18, 842 (1994).

(15) J. S. Paek, J. T. Kim and M. S. Gong, Polymer (Korea), 19, 561(1995).

(16) O. Kim and M. S. Gong, Polymer (Korea), 22, 446 (1998).

(17) C. W. Lee and M. S. Gong, Macromol. Res., 11, 322 (2003).

(18) J. S. Paek and M. S. Gong, Korean J. Mater. Res., 5, 715 (1995).

(19) Y. Sakai, Y. Sadaoka, and H. Hukumoto, Sens. Actuators, 13, 243 (1988).

(20) Y. Sakai, M. Matsuguchi, and T. Hurukawa, Sens. Actuators $B, 66,135$ (2000).

(21) M. S. Gong, S. W. Joo, and B. K. Choi, J. Mater. Chem. 12, 902 (2002).

(22) C. W. Lee, O. Kim, and M. S. Gong, J. Appl. Polym. Sci. 89, 1062 (2003).

(23) C. W. Lee and M. S. Gong, Sens. Actuators B, 11, 322 (2003).

(24) J. Jang, J. Bae, and D. Kang, J. Appl. Polym. Sci. 82, 2310 (2001).

(25) T. M. Kim, J. K. Park. R. Y. Lee, and M. S. Gong, Korean J. Mater Res. 3, 598 (1993). 